\title{
Predicción del índice de calidad en canales bovinas para mercado chileno de elite mediante modelación matemática ${ }^{\#}$
}

\author{
Prediction of a quality index of bovine carcasses for elite Chilean market using a mathematical model \\ J Campos*, P González, M Doussoulin, M Tima, PA Williams \\ Departamento de Producción Animal, Facultad de Agronomía, Universidad de Concepción, Chillán, Concepción, Chile.
}

\begin{abstract}
SUMMARY
The Chilean beef sector has to satisfy the international demand of beef with homogenous and high quality products. Since quality is a subjective parameter, it is necessary to find the variables to objectively characterize the carcasses. Further development of mathematical models as tools for better prediction and classification of beef quality is needed. The aim of this study was to predict beef quality for the Chilean market, using the data generated by the programme "Programa de Desarrollo de Proveedores (PDP)" implemented by Carnes Ñuble S.A. between 1999 and 2003 . Twelve variables were generated. Five of them received a score and its addition resulted in the quality index. This index allowed the classification of the meats as "common" and "special", being the last group the one used by the "Pampaverde" ${ }^{\circledR}$ brand. The data were studied through a descriptive analysis, as well as correlation analysis, considering the variables that contributed more to the total score. Moreover, a multiple lineal regression analysis was conducted. Using this information, a model was built to predict Chilean beef quality. The model was evaluated and validated using data from 2002.
\end{abstract}

Palabras clave: canal bovina, calidad de carne, modelo predictivo. Key words: bovine carcass, meat quality, predictive model.

\section{INTRODUCCIÓN}

El consumo de carne bovina en Chile ha disminuido debido a cambios en los gustos y patrones de preferencias de los consumidores, como también sucede en países europeos (Piggott y col 1996, Rickertsen 1996). Uno de los factores más relevantes en este cambio es la preocupación de los consumidores por la calidad de los productos y su efecto en la salud (USDA/ERS, 2002, Schnettler y col 2004). Los atributos homogeneidad del producto y cuán saludables son (Padilla y col 2007) pueden ser evaluados asignándoles etiquetas de certificación de calidad (Oyarzún y Tartanac 2002). El sello de calidad puede facilitar al consumidor el proceso de compra e incidir en su preferencia (Fotopoulos y Krystallis 2003). En Chile existe preferencia por carnes magras, con cortes de buen tamaño y tiernas, lo cual se considera como carnes especiales según lo mencionado por Schnettler y col (2004) que coincide con lo reportado por Yang y col (2006) para las preferencias de consumidores de fuera del país. Un factor que debe considerarse es que la demanda por carne está además influenciada por el poder adquisitivo del consumidor, por lo tanto serían los consumidores con mayores ingresos los que podrían acceder a productos certificados (Padilla y col 2007).

Aceptado: 03.12.2008.

\# $\quad$ Financiado por Proyecto DIUC No 206.121.011-1.0 U de C 2006.

* Casilla 537, Chillán, Chile; jcamposp@udec.cl
En Chile, el consumidor de carne bovina ha ido evolucionando en su preferencia, categorizando los alimentos según la seguridad e higiene del producto, sabor, frescura, pureza (Schnettler y col 2004), tipo de corte (que éstos sean magros, sin hueso) y valorando características como terneza, sabor y jugosidad de la carne (Magofke 1992).

Para lograr la homogeneidad del producto carne, como factor de calidad, es necesario identificar qué tan comparable es la carne producida a lo largo del país, considerando en este punto que exista estabilidad en el producto y que cumpla con un estándar mínimo. La homogeneidad del producto estará dada por la raza del animal que se faene y el sistema productivo que se utilice para producir carne. La genética más usada en los sistemas de producción de carne bovina chilenos es el ganado de doble propósito, en tanto la producción de carne con razas especializadas representa sólo entre el 25 a 30\% de la masa ganadera total (Becerra y Klee 1991, Esnaola y Poblete 1993, Amesti 2000).

La apertura económica de Chile al mercado internacional de carnes precisa una mayor integración en la producción y comercialización de la carne bovina, para así aumentar la competitividad e incrementar su participación en éste (Saravia 1996). La planta faenadora de animales Carnes Ñuble S.A. entre los años 1999 y 2003 implementó un programa de desarrollo de proveedores (PDP), a través del cual logró introducir al mercado nacional una marca propia, conocida como "Pampaverde" ${ }^{\circledR}$, que genera productos homogéneos y con certificación de calidad, permitiendo agregar valor y diferenciación 
de sus productos frente a la competencia (Ávila 2002). Además, en el mercado chileno se cuenta con otras marcas de carne con certificación de calidad, entre estas "Carne a Punto" ${ }^{\circledR}$, producida por Frigorífico Temuco.

Dado que el concepto "calidad" de un producto es subjetivo, es necesario conocer parámetros que permitan hacerlo objetivo. Para identificar parámetros productivos que incidan en la calidad de la carne y permitan tomar decisiones, existen diversas metodologías. Entre éstas se destacan los modelos matemáticos que permiten predecir el comportamiento de los sistemas productivos frente a cambios en las alternativas de manejo, usando programas computacionales (Webster 2000). Los modelos para que sean confiables y aplicables requieren de un proceso de evaluación y validación, para lo cual es preciso compararlos con observaciones reales obtenidas in vivo (Steel y Torrie 1995).

El presente trabajo tiene como objetivo predecir la calidad de carne bovina, para mercado nacional, a través de la construcción de un modelo basado en parámetros de calidad.

\section{MATERIAL Y MÉTODOS}

El estudio se realizó en base a los registros oficiales de la planta faenadora Carnes Ñuble S.A. del año 2001, que corresponden a 7.300 bovinos provenientes de 40 productores de la Zona Centro Sur de Chile, miembros del PDP Carnes Nuble S.A.

Las variables utilizadas fueron generadas por la propia industria obtenidas de las planillas oficiales de registro, las cuales fueron medidas por certificadores externos a la planta y corresponden a:

PESO VIVO (KG)

Peso promedio del animal vivo (promedio por lote).

PESO DE LA CANAL (KG)

Peso del animal luego de ser faenado y antes del desposte, en canal caliente, según INN (1993) y sin la grasa de riñón.

RENDIMIENTO (\%)

Rendimiento centesimal (Porte 1994).

CLASE

Los animales se clasificaron en novillos (NOV) y vaquillas (VAQ).

\section{TIPIFICACIÓN}

La tipificación se realizó de acuerdo a la NCh 1306.Of 93. Las canales se tipificaron como: categoría V (3) y categoría A (2), apareciendo en paréntesis el puntaje entregado por la planta.
FENOTIPO

Se determinó en base a observaciones de las características fenotípicas más relevantes del animal, confeccionándose los siguientes grupos: Holstein Friesian (HF), Angus rojo o negro (AN), Hereford (HE), Doble propósito (DP) incluyendo a Overo Colorado y Overo Negro; Híbrido de carne (HC) incluyendo a Hereford $x$ Overo Negro; Hereford x Angus; Hereford x Overo Colorado; Angus x Overo y Simmental x Overo.

\section{CONFORMACIÓN}

Las canales se clasificaron como buena (3), regular (2) y mala (0), apareciendo en paréntesis el puntaje entregado por la planta.

\section{ÁREA DEL OJO DEL LOMO (AOL) (PULG $\left.{ }^{2}\right)$}

Se midió a la altura de la décima costilla, en frío, al momento de obtener los cuartos de las canales, utilizando la metodología propuesta por Porte (Porte 1994). Estableciendo cuatro categorías para NOV: menor que 14; 14 a 16; 16 a 17 y mayor que 17; y cuatro para VAQ: menor que 12; 12 a 14; 14 a 15 y mayor que 15 . Asignándole a cada categoría, tanto en NOV como en VAQ: -1; 0; 3 y 8 puntos, respectivamente.

\section{ESPESOR DE GRASA DORSAL (EGD) (CM)}

La medición se realizó a través de una regla graduada en milímetros $(\mathrm{mm})$, que se ubicó en el borde de la capa de grasa que cubre el AOL, agrupándolas en cinco categorías: menor a 0,20 ; de 0,21 a 0,40 ; de 0,41 a 0,70 ; de 0,71 a 1,00 y mayor que 1 asignándoles $0 ; 4 ; 1 ; 0$ y -8 puntos, respectivamente.

\section{MARMOLEO (MAR)}

Se calificó por simple observación de la distribución y grosor de las vetas de grasa en el AOL, generando cinco grados distintos de intensidad, desde la categoría grado uno (bajo) hasta categoría grado cinco (excesivo), siendo el puntaje otorgado a cada categoría de: $6 ; 7 ; 8 ; 2$ y 0 puntos, respectivamente.

\section{ÍNDICE DE CALIDAD (IC)}

Sumatoria de los puntajes obtenidos de las canales bovinas de acuerdo a las variables tipificación, conformación, MAR, AOL y EGD, cuyos valores fueron asignados por la planta. Esta variable permitió a la empresa definir calidad, ya que clasifica a las canales en "Corriente" y "Especial", siendo esta última definida por sobre los 18 puntos. Las canales clasificadas como "Especial" son las que abastecen de materia prima a la marca "Pampaverde" ${ }^{\circledR}$, destinada a mercado nacional. 


\section{ANÁLISIS ESTADÍSTICO}

Se realizó un análisis exploratorio mediante estadística descriptiva de los registros oficiales de la planta del año 2001 (7.300) para NOV y VAQ. Posteriormente, se calculó la matriz de correlaciones entre las variables tipificación, conformación, MAR, AOL, EGD e IC; para realizar este análisis se utilizó el coeficiente de rangos de Spearman (Steel y Torrie 1995, Webster 2000).

Las variables que se utilizaron en el modelo de regresión lineal múltiple fueron aquellas que presentaron una alta correlación $(\mathrm{P}<0,01)$ con la variable dependiente (IC). Se confeccionó un modelo con toda la masa bovina estudiada y modelos agrupados por clase y fenotipo.

Con los ocho modelos generados: dos por clase, cinco por fenotipos y masa total, se realizó una comparación mediante un análisis de varianza (ANDEVA), con un nivel de significancia del $5 \%$, para lo cual se generaron muestras de 50 datos por modelo. Con respecto a la verificación de los supuestos de normalidad de los residuos y de homogeneidad de las varianzas del modelo se utilizaron los test de Shapiro Wilks modificados y Bartlet, además, para desechar problemas de multicolinealidad en el modelo se utilizó la matriz de correlación entre las variables. Para mostrar la validación del modelo se utilizó un gráfico de puntos. Para medir la capacidad de predicción del modelo se utilizó la prueba de exactitud de Freese (Freese 1960, Barrales y col 2004), con un 5\% de significancia; para ello se utilizó una muestra de 100 datos elegidos al azar de los registros oficiales de la planta faenadora del año 2002, de los miembros del PDP Carnes Ñuble S.A.

El análisis de los datos se realizó mediante el software InfoStat, versión 2004, y actualizaciones de la Universidad de Córdoba, Argentina, y para el manejo de los datos y confección de gráficos se utilizó el software Excel de Microsoft Office.

\section{RESULTADOS Y DISCUSIÓN}

Del análisis descriptivo se puede inferir que, para las categorías de los animales faenados en la planta, se pudo apreciar un mayor beneficio de NOV, independiente del fenotipo. El ganado faenado correspondió principalmente a $\mathrm{HC}$ seguido por DP, los que en sumatoria representaron el $65 \%$ del total de animales, y tan sólo un $24 \%$ de ellos correspondieron a razas de carne. $\mathrm{Al}$ analizar los resultados por fenotipos de los animales faenados, se puede apreciar la poca especialización que existe de la producción de carne. El fenotipo HE es el principal representante de las razas especializadas en carne, ocupando el 55\% de la masa ganadera de aptitud cárnica. El resto está constituido por $\mathrm{HC}$ y por el fenotipo AN, teniendo este último una participación creciente en los predios ganaderos (Anrique 1993, Navarro 2001).

Los pesos vivos promedios (Media $\pm \mathrm{DE}$ ) fueron similares entre todos los fenotipos tanto para NOV como para VAQ, correspondiendo a $441 \pm 49$ y $367 \pm 38 \mathrm{~kg}$, respectivamente. El peso promedio de las canales de NOV y VAQ fue de $239 \pm 28$ y $199 \pm 24 \mathrm{~kg}$, respectivamente. Troncoso (1982) y González y col (1991) indican que el peso promedio de las canales de VAQ es un $17 \%$ menor que el de las de NOV, lo que concuerda con los resultados encontrados en este estudio, donde el peso promedio de las canales para VAQ fue un 16,7\% inferior al de los NOV. El menor peso de las canales, obtenido en este estudio, se puede asociar a la creciente demanda por consumir carnes con bajo contenido de grasa, las que provienen de animales jóvenes y con menor peso de beneficio, provocando una mayor preocupación por evitar el sobreengrasamiento de los animales (González y col 1991). El sobreengrasamiento está siendo castigado en el precio a nivel de las transacciones comerciales, lo que se puede observar en los puntajes entregados a las distintas variables por la planta.

El rendimiento centesimal de NOV y VAQ varió entre 52 y $55 \%$, con una desviación estándar de un $2 \%$ para ambos casos.

El IC presentó mayores coeficientes de correlación con las variables MAR, AOL y EGD, siendo ellas significativas $(\mathrm{P}<0,01)$ (cuadro 1). La variable tipificación presentó coeficientes de correlación mayores con MAR, AOL y EGD. Este último índice de correlación positivo podría deberse al hecho de que la masa ganadera presentó características homogéneas, como, por ejemplo, animales tipificados "V" en un $95 \%$, con conformación buena a regular un $98 \%$ y un grado de MAR 2 a 3 en un $87 \%$.

Del análisis de correlación se pueden inferir cuáles son las variables que más influyen en el índice de calidad (IC) que condiciona que la carne sea clasificada para ser un producto "Pampaverde" ${ }^{\circledR}$. Por tanto, las variables MAR, AOL y EGD presentaron un mayor coeficiente de correlación $(\mathrm{P}<0,01)$ y se utilizaron para generar los modelos

Cuadro 1. Matriz de coeficientes de correlación entre las variables: tipificación (TIP), conformación (CONF), marmoreo (MAR), área del ojo del lomo (AOL), espesor de grasa dorsal (EGD) y puntaje de planta (IC).

Matrix of correlation coefficient index between variables: tipification (TIP), conformation (CONF), marbling (MAR), sirloin eye muscle area (AOL), backfat thickness (EGD) and slaughter score (IC) for the total cattle group.

\begin{tabular}{lllllll}
\hline & TIP & CONF & MAR & AOL & EGD & IC \\
\hline TIP & 1 & $0,57^{*}$ & $0,50^{*}$ & $0,46^{*}$ & $0,53^{*}$ & $0,42^{*}$ \\
CONF & & 1 & 0,26 & $0,39^{*}$ & 0,27 & $0,39^{*}$ \\
MAR & & & 1 & 0,17 & 0,35 & $0,69^{* *}$ \\
AOL & & & & 1 & 0,21 & $0,68^{* *}$ \\
EGD & & & & & 1 & $0,53^{* *}$ \\
IC & & & & & & 1 \\
\hline
\end{tabular}

* Indica correlación significativa $(\mathrm{P}<0,05)$.

** Indica correlación significativa $(\mathrm{P}<0,01)$. 
de regresión, debido a que son capaces de explicar hasta el 98\% del IC. Lo anterior concuerda con lo planteado por Yang y col (2006) que indican que las variables MAR y EGD le imprimen sabor, jugosidad y terneza a la carne.

A través del análisis de varianza que se realizó a los puntajes generados por cada uno de los modelos, se estableció que no existe diferencia significativa $(\mathrm{P}>0,05)$ entre ellos. Por lo que para la validación del modelo se utilizó la ecuación generada para la masa bovina total y que correspondió a:

$$
\mathrm{IC}=4,83+1,01 \mathrm{MAR}+1,07 \mathrm{AOL}+1,03 \mathrm{EGD}
$$

En la figura 1 se observa la comparación entre el promedio y la desviación estándar mensual del IC generado por el modelo versus el puntaje real obtenido de las planillas oficiales de registro de la planta del año 2001, donde se observa que el rango de error presentado por el modelo es mínimo frente al puntaje real.

En la evaluación del modelo propuesto para predecir calidad de carne, al aplicarse la prueba de exactitud de Freese, se obtuvo un valor de $\mathrm{X}^{2}=56,2$, el cual se comparó con el valor tabular de $\mathrm{X}_{(99,0,05)}^{2}=123,22$, lo que permite validar la capacidad predictiva del modelo.

Si a las variables MAR, AOL y EGD se les cambiara el puntaje asignado en función de las preferencias de consumidores de otros mercados, podría predecirse qué porcentaje de animales podría ser comercializado en ese mercado particular. Además, la identificación de las variables que más influyen en la calidad de carne lleva a pensar que sería posible, aplicando tecnologías, poder medir estas variables ante mortem, lo que permitiría al productor o a los compradores de ganado tener una estimación de la calidad de su carne.

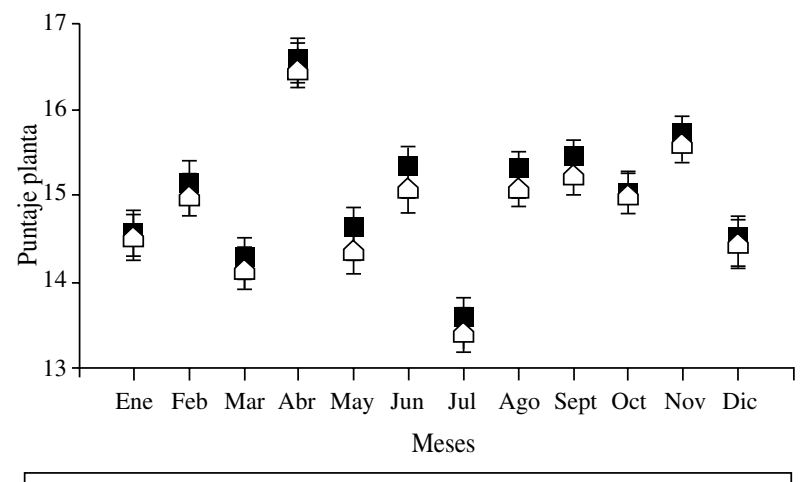

Puntaje de planta real $\triangle$ Puntaje de planta generado por modelo

Figura 1. Comparación del promedio y desviaciones estándar mensuales del puntaje de planta generado por la ecuación de regresión total versus el puntaje real obtenido de las planillas de registro del año 2001.

Comparison of the mean and standard deviation by month of the slaughter score generated by the total regression equation versus the real slaughter score obtained by the slaughter records for the year 2001 .
Dado que el modelo propuesto según la prueba de exactitud de Freese permite predecir el IC, es que sería válido determinar las variables MAR, AOL y EGD para predecir si el animal faenado puede ser comercializado como carne especial.

En conclusión, es posible predecir el IC de carne especial bovina utilizando un modelo matemático.

\section{RESUMEN}

El sector pecuario chileno ha tenido que responder a las demandas internacionales con productos homogéneos y de gran calidad. Como la calidad es un parámetro subjetivo, se ha buscado la manera de parametrizarla, para lo cual se debe disponer de diferentes herramientas, como los modelos matemáticos. El objetivo del estudio fue predecir la calidad de carne bovina para mercado nacional, usando la información generada por el Programa de Desarrollo de Proveedores (PDP) que implementó Carnes Ñuble entre los años 1999 al 2003. Se generaron 12 variables, de las cuales a cinco de ellas se les asignaron puntajes, que al sumarlos originaron el puntaje de planta que corresponde al índice de calidad. Este índice permitió clasificar las canales bovinas en "Corrientes" y "Especial", siendo estas últimas las que abastecen de materia prima a la marca "Pampaverde" ${ }^{\circledR}$. Los datos se estudiaron mediante un análisis descriptivo también se realizó un análisis de correlación con las variables que más aportan al puntaje de planta. Además, se realizó un análisis de regresión lineal múltiple. Con esta información, se construyó un modelo que permite predecir la calidad de la carne para mercado nacional. Este modelo fue validado con datos del año 2002.

\section{REFERENCIAS}

Amesti E. de. 2000. Carne bovina, panorama mundial y situación en Chile. Agroeconómico 58, 16-21.

Anrique R. 1993. Rol de las razas en la producción de carne bovina. Agroeconómico 14, 20-24.

Ávila JI. 2002. Diseño e implementación del programa de desarrollo de proveedores Carnes Nuble S.A. Memoria de título, Facultad de Agronomía Universidad de Concepción, Chillán, Chile.

Barrales L, I Peña, P Fernández. 2004. Validación de modelos: un enfoque aplicado. Agric Téc 64, 1, 66-73.

Becerra L, G Klee. 1991. Sistemas de producción con novillos Holandeses para el valle regado. INIA Quilamapu 48, 17-21.

Esnaola V, C Poblete. 1993. Comercialización de la carne. Agroeconómico $15,10-17$.

Fotopoulus C, A Krystallis. 2003. Quality labels as a marketing advantage: the case of the "PDO Zaragoza" apples in the Greek market. Eur J Marketing 37, 1350-1374.

Freese F. 1960. Testing accuracy. For Sci 6, 139-145.

González F, R Martínez, C Bravo. 1991. Relaciones de peso en canales bovinas cuarteadas a nivel del $9^{\circ}$ espacio intercostal. Agro-Ciencia 7, 143-147.

MacClure F. 2004. Comportamiento del mercado nacional e internacional de la carne bovina: Análisis de la engorda y perspectivas de Chile para exportación. Memoria de título, Facultad de Agronomía e Ingeniería Forestal, Pontificia Universidad Católica de Chile, Santiago, Chile.

Magofke JC. 1992. Caracterización de algunas razas bovinas de carne. Arch Prod Anim 17, 3-22.

Navarro J. 2001. Producción de carnes de calidad certificada. Agroeconómico 64, 42-45.

Oyarzún M, F Tartanac. 2002. Estudio sobre los principales tipos de sellos de calidad en alimentos a nivel mundial. Estado actual y perspectivas de los sellos de calidad en productos alimenticios de la agroindustria rural en América Latina. Santiago, Chile. 
Padilla C, P Villalobos, A Spiller, G Henry. 2007. Consumer preference and willingness to pay for an officially certified quality label: Implications for traditional food producers. Agric Tec 67, 300-308.

Piggott N, J Chalfant, J Alston, G Griffith. 1996. Demand response to advertising in the Australian meat industry. Am J Agric Econ 71, 253-261.

Porte E. 1994. Rendimientos y cortes de carne. En: Porte E (ed). Producción de carne bovina. $4^{\mathrm{a}} \mathrm{ed}$. Editorial Universitaria, Santiago, Chile, Pp 299-311.

Rickertsen K.1996. Structural change and demand for meta and fish in Norway. Eur Rev Agric Econ 23, 316-330.

Saravia A. 1996. La importancia de las cadenas agroalimentarias. En:

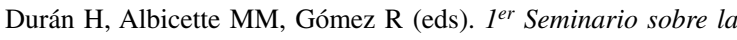
importancia de la integración en la cadena cárnica. Plan agropecuario. Montevideo, Uruguay, Pp 17-29.

Schnettler B, O Manquilef, H Miranda. 2004. Atributos valorados en la selección de carne bovina por el consumidor de supermercados de Temuco, IX Región de Chile. Ciencia Inv Agr 31, 2, 91-100.
Schnettler B, Manquilef, H Miranda. 2006. Hábitos de consumo de carne bovina en Temuco, IX Región de Chile. IDESIA 24, 15-23.

Steel R, J Torrie. 1995. Bioestadística, principios y procedimientos. $2^{\mathrm{a}} \mathrm{ed}$ Editorial Latinoamericana, México D.F., México.

Troncoso JE. 1982. Calificación objetiva de canales de hembras (vacas y vaquillas) de Doble propósito Overo Europeo (ONE) y Overo Colorado Alemán (OCA) I Parte: Índice de carnosidad bruta. Memoria de título, Fac. Ciencias Agr. Pecuarias y Forestales, Universidad de Chile, Santiago, Chile.

USDA. Economic Research Service. 2002. Changing consumer demands create opportunities for U.S. food system. Food Reviews 25, 19-22.

Webster A. 2000. Estadística aplicada a los negocios y la economía. $3^{\mathrm{a}}$ ed. Ed McGraw-Hill, Bogotá, Colombia.

Yang XJ, E Albrecht, K Ender, RQ Zhao, J Wegner. 2006. Computer image analysis of intramuscular adipocytes and marbling in the longissimus muscle of cattle. J Anim Sci 84, 3251-3258. 